\title{
Research Paper \\ Compliance to Universal Design Criteria in Nursing Homes of Tehran
}

Morteza Nasiri ${ }^{1}$, Mahshid Foroughan $^{2}$, Vahid Rashedi ${ }^{2}$ Asghar Makarem¹, Bahram Jafari Mourjan ${ }^{1}$

1. Department of Rehabilitation Managment, University of Social Welfare and Rehabilitation Sciences, Tehran, Iran.

2. Iranian Research Centre on Ageing, University of Social Welfare and Rehabilitation Sciences, Tehran, Iran.

Crtation: Nasiri M, Foroughan M, Rashedi V, Makarem A, Jafari Mourjan B. [Compliance to Universal Design Criteria in Nursing Homes of Tehran (Persian)]. Iranain Journal of Ageing. 2016; 11(2):340-347. http://dx.crossref.org/10.21859/sija-1102340

http://dx.crossref.org/10.21859/sija-1102340

Received: 12 Mar. 2016 Accepted: 28 May. 2016

Key words:

Universal design,

Nursing home,

Older adults

\section{ABSTRACT}

Objectives There has been a rapid growth in the number of older people residing in the nursing homes. Therefore, it is highly important to prepare the physical milieus that are responsive to their physical, psychosocial, and spiritual needs. The universal design implies designing the products and environments in a manner that it is usable by all people, to the greatest extent possible, without the need for further adaptation. The aim of this study was to evaluate the conformity of the physical milieus of the nursing homes in Tehran to the universal design criteria.

Methods \& Materials This was a cross-sectional descriptive study. All the nursing homes for older people located in the city of Tehran during the year 2015 were evaluated using the Nursing Home Physical Fitness Checklist, which consisted of 12 subscales and 70 items. Psychometric properties of this tool were evaluated and confirmed prior to the experiment. The collected data was analyzed using the measures of central tendency.

Results Majority of nursing homes in terms of kitchen, management section, laundry room, heating and cooling conditions, main entrance, treatment room, and the toilets were in conformance with the universal design criteria, while only one-third of the homes had extinguishers. The criteria was not adequately met in terms of the items of available chapel and library, staff restroom, living room, and dining hall.

Conclusion The findings of this study showed that the majority of nursing homes evaluated did not follow the universal design criteria. Therefore, providing the proper guidelines and policies to promote the universal design observance in nursing homes is considered as a major necessity.

\section{* Corresponding Author:}

Mahshid Foroughan, PhD

Address: Iranian Research Centre on Ageing, University of Social Welfare and Rehabilitation Sciences, Kodakyar Ave., Daneshjoo Blvd., Evin, Tehran, Iran. Tel: +98 (21) 22180077

E-mail: m_foroughan@yahoo.com 


\title{
وضعيت رعايت اصول طراحى فراتير در آسايشَّاههاى سالمندان شهر تهران
}

\author{
مرتضى نصيرى'؛ "مهشيد فروغان'، وحيد راشدى'، اصغر مكارم'، بهرام جعفرىمورجان' \\ 1- كروه مديريت توانبخشى، دانشعاه علوم بهزيستى و توانبخشى، تهران، ايران.

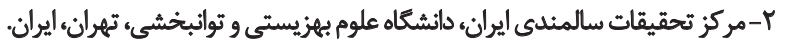

\begin{abstract}
21,

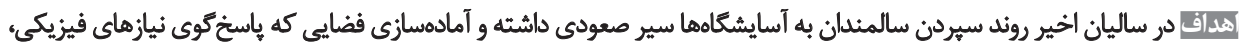

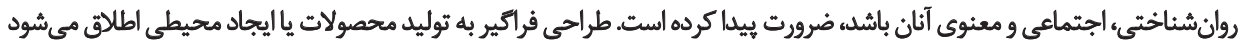

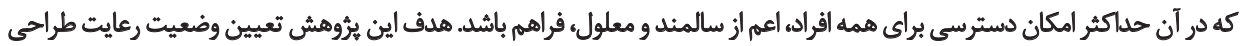

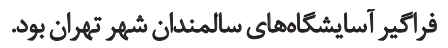

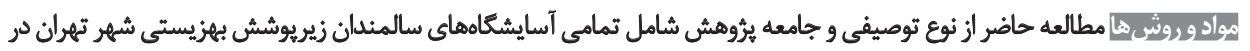

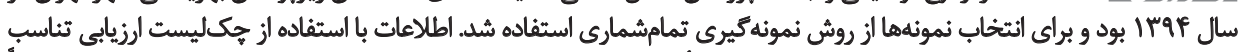

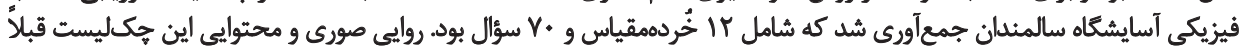

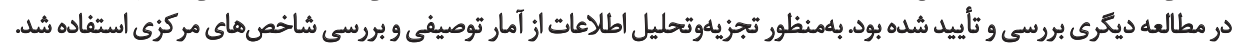

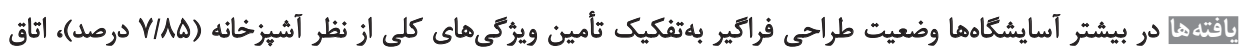

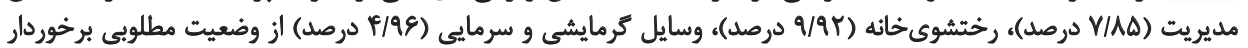

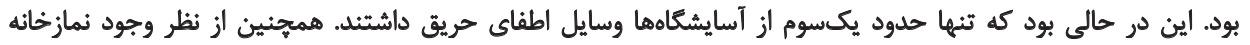

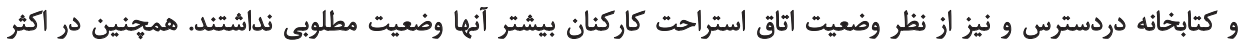

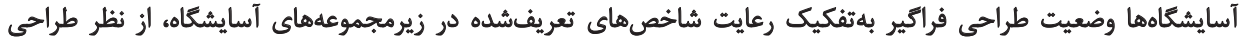

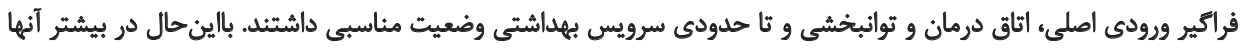

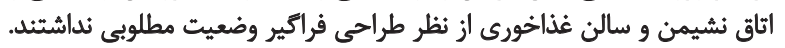

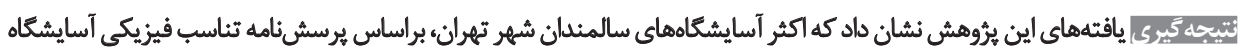

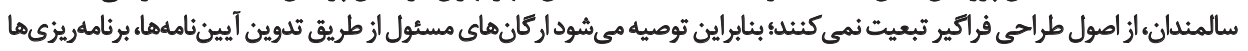

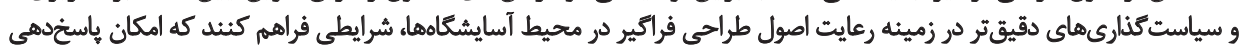

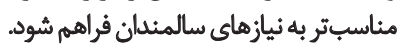

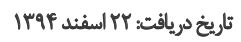

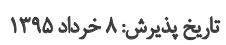

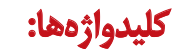

طراحي فراكير، آسايشغاه، سالمند فراحن

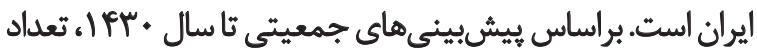

مقدمه

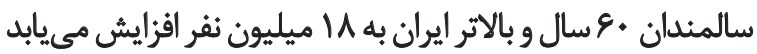

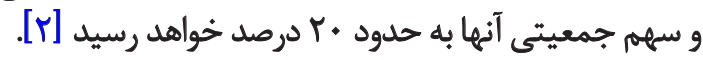

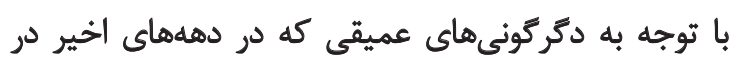

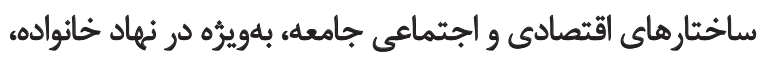

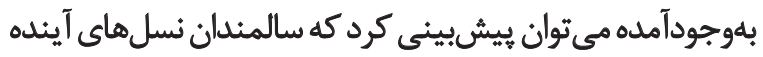

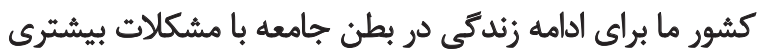

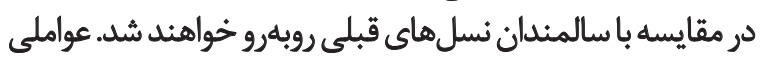
مانند تغيير در ساختار خانواده (از گَسترده به هستهاي) و افزايش

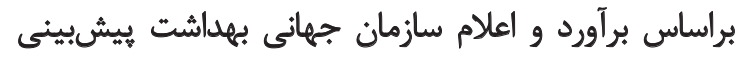

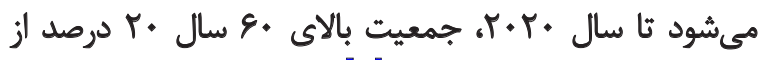

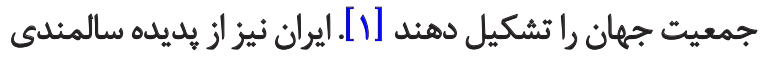

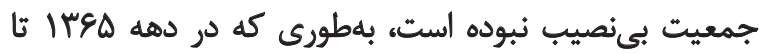
ITVD

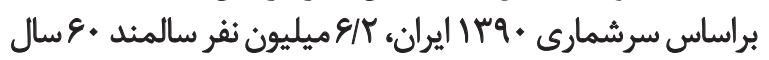

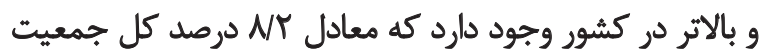


فيزيكى و اجتماعى محيط زندكى سالمندان نيز بر ساز كارى آنان

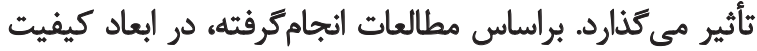

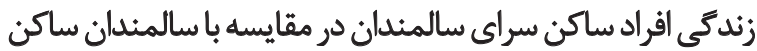

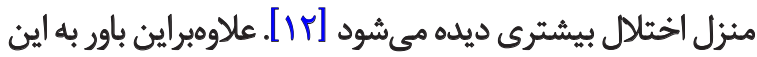

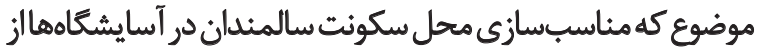

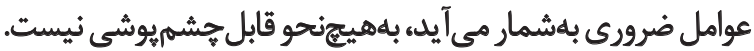

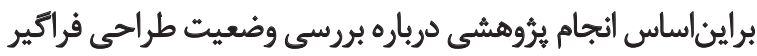

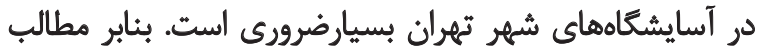

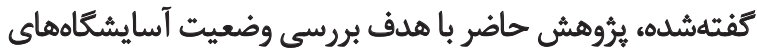

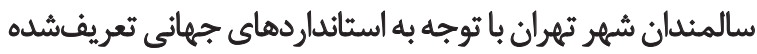

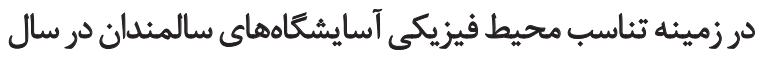

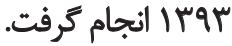

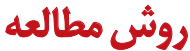

اين تحقيق به روش توصيفى انجام شد و جامعه آمارى آن را

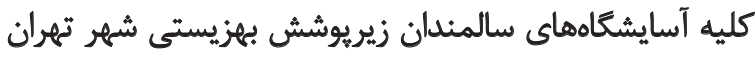

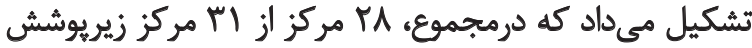

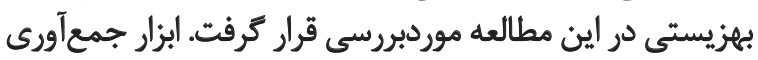

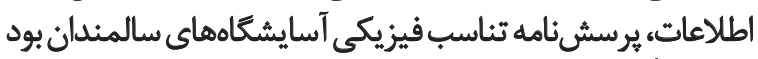

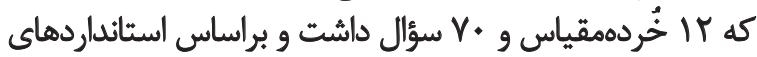

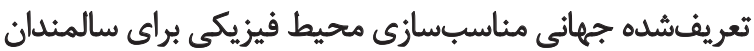

با استفاده از مستندات علمى موجود تهيه شده بودي ميكي

براى تعيين روايى صورى، اين ابزار به • ب نفر از سالمندان

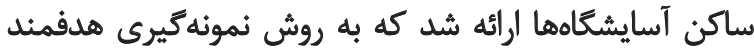

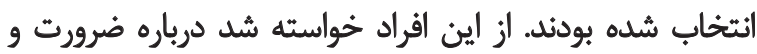

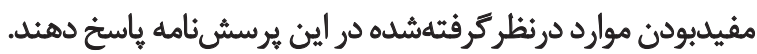

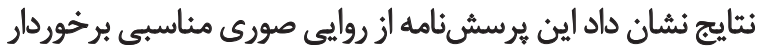

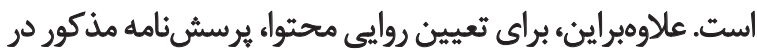

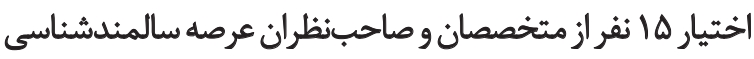

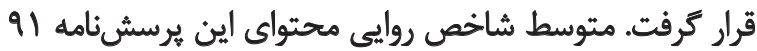

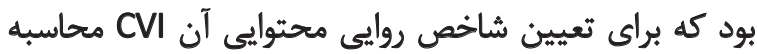

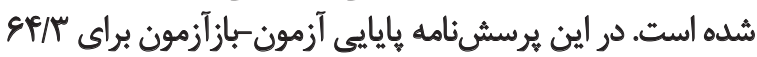

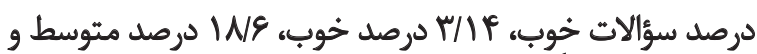
T/A درصد نسبتاً ضعيف تزارش ترديده است

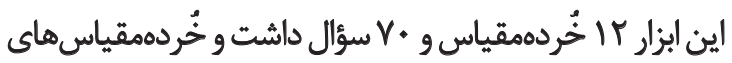

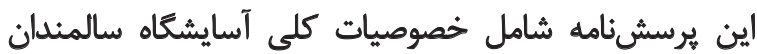

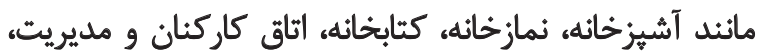

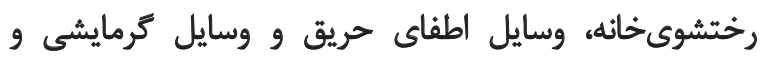

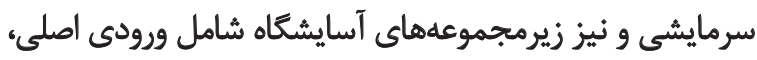

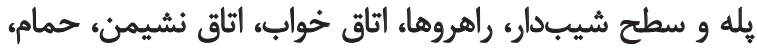

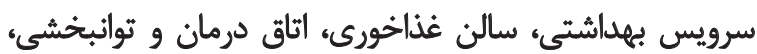

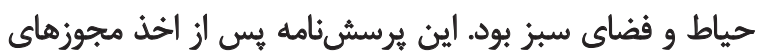
لازم و با مراجعه به تمامى مراكز سالمندى و تكميل تمام آيتمها آنذاي
عواملى نظير زندكى آيارتمانى، مهاجرت براى بهببود معاش، تغيير

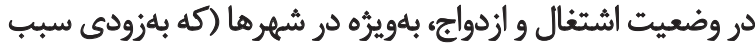

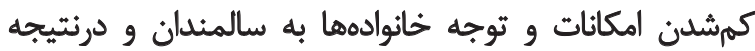

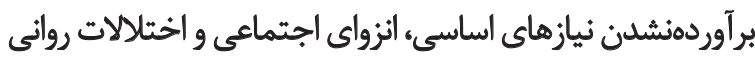

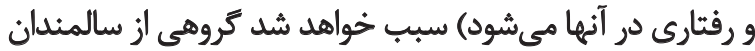

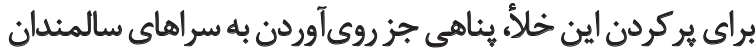
ئيشروى خود نبينيند.

مطالعات نشان مى دهد كه استفاده از آسايشعاههاى سالمندان

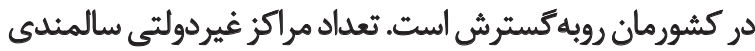

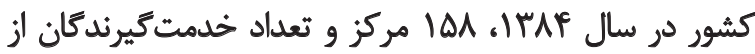

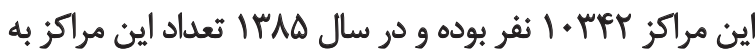

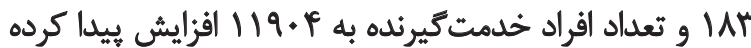

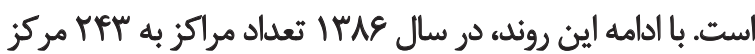
با IOYVD ا در طول فرايند سالمندى، يك فرد بالغ سالم ممكن است به فيه

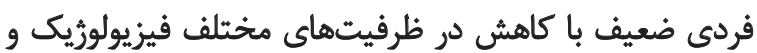

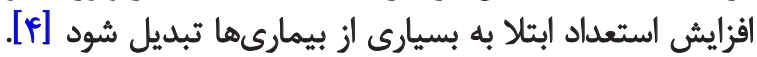

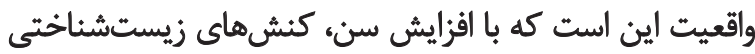

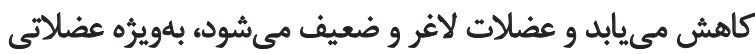

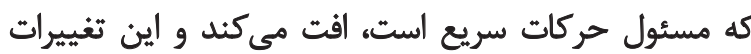

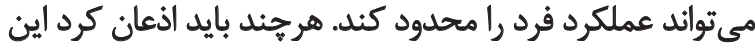

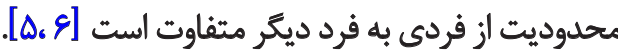

بررسىها نشان مى دهد كه بيش از نيمى از سالمندان در انجام

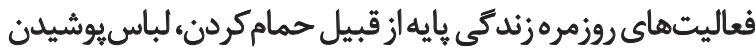

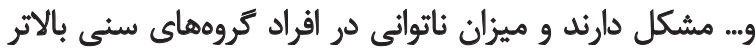

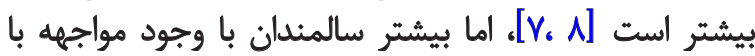

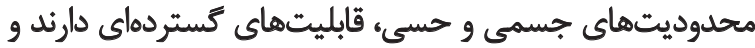

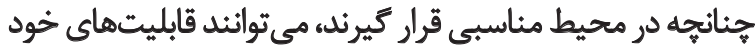

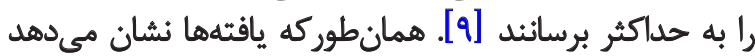

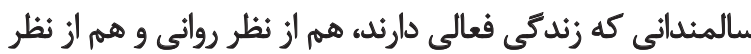

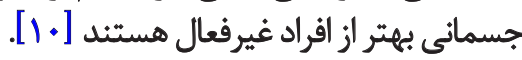
يُروهشعران معتقدند فعاليت فيزيكى در بسيارى از سالمندان

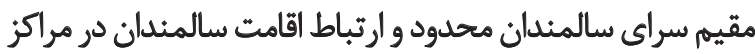

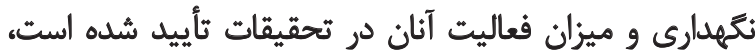

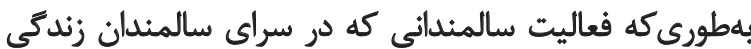

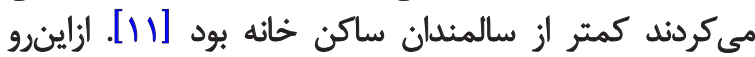

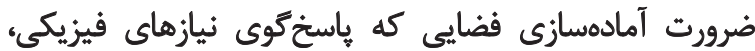

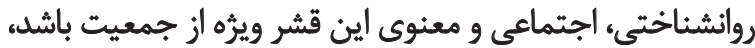

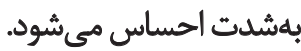
با توجه به ايثكه در ساليان اخير روند سيردن سالمندان به

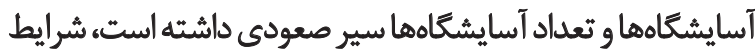




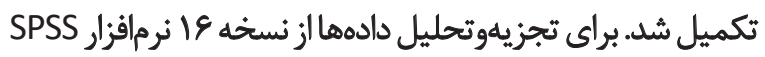

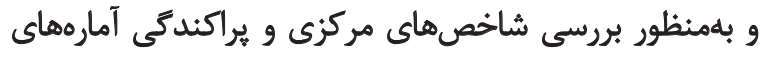
توصيفى استفاده شد.

براساس نتايج بهدست أمدهاز إين يُوهش، بيشتر آسايشكاههاى

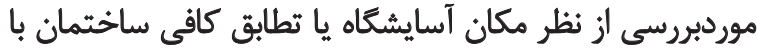

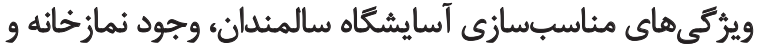

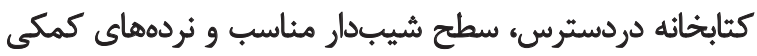

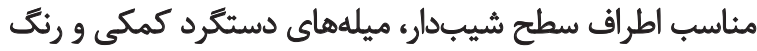

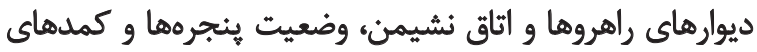

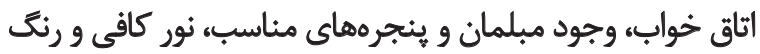

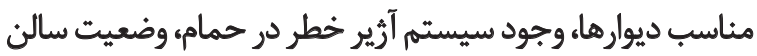

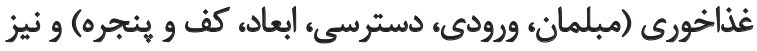

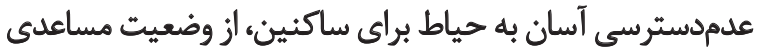
براى سالمندان ساكن آسايشكاه برخوردار نبودي يافتهها حاكى از آن است كه وضعيت نامناسب سالن

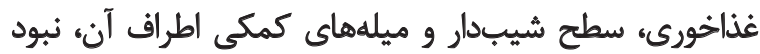

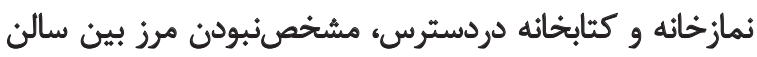

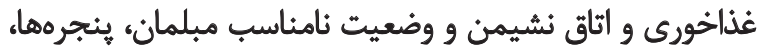

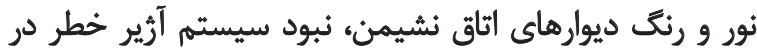

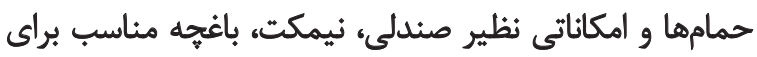

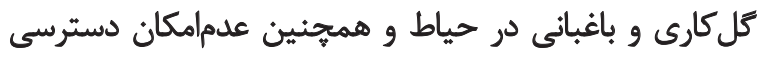

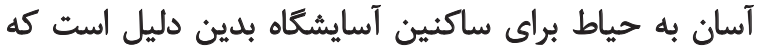

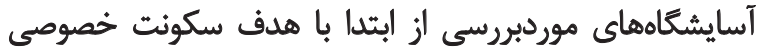

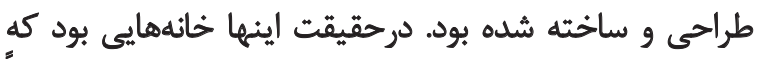

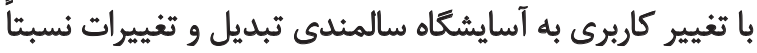

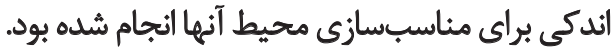
نوذرى و همكارانش در مطالعه خود نشان دادند هيجيك از ملازيط

\section{ياقتهها}

درباره وضعيت طراحى فراكير آسايشكاههاى سالمندان شهر

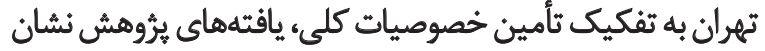

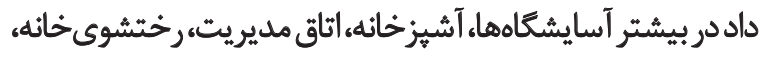

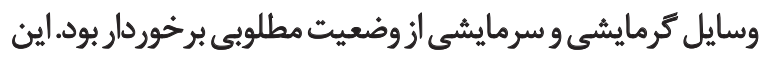

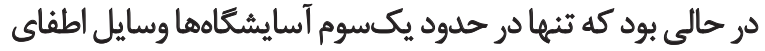

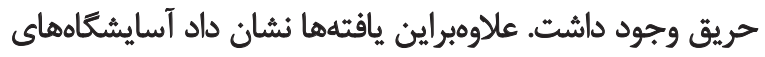

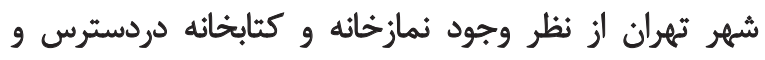

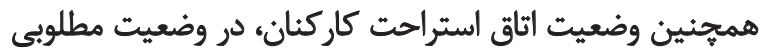

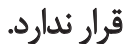

در زمينه وضعيت طراحى فراتير آسايشكاههاى سالمندان

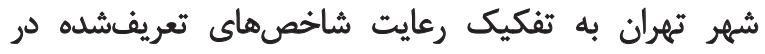

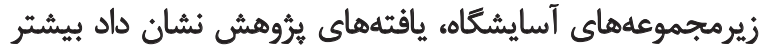

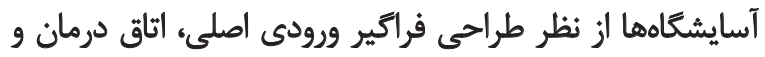

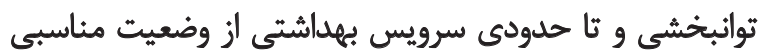

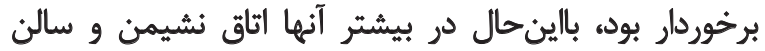

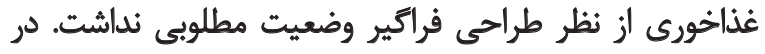

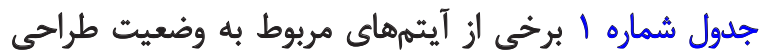

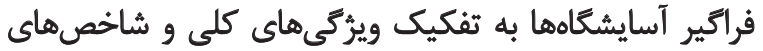

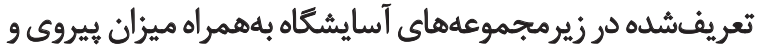
عدمييروى از اصول طراحى فراكير نشان داده شده است.

جدول ا. وضعيت طراحى فراكير آسايشكاههاى شهر تهران به تفكيك ويرّكى هاى كلى و شاخصهاى تعريفشده در زيرمجموعههاى آسايشكاه.

\begin{tabular}{|c|c|c|c|c|c|c|}
\hline \multicolumn{7}{|c|}{ وضعيت طراحي فراكير آسايشعَاهها } \\
\hline \multicolumn{2}{|c|}{ عدم رعايت } & \multicolumn{2}{|c|}{ رايت } & & & \\
\hline درصد & تعداد & درصد & تعداد & & & \\
\hline mis & 1 & $q \varepsilon / p$ & rV & وسايل سرمايشى و كرمايشى & & \\
\hline$e * / \pi$ & M & $r \Delta / v$ & 1. & وسايل اطفاي حريق & & \\
\hline $\mid f / \mu$ & $f$ & $\Lambda \otimes / V$ & rf & أشيزخائه & & \\
\hline $\mathrm{V} / \mathrm{I}$ & r & $97 / 9$ & re & رختشوى خانه & التاق كاركثان و مديريت & ويرُكى هاى كلى \\
\hline$\varepsilon \cdot / V$ & iv & $r q / r$ & 11 & أتاق استر احت كاركنان & & \\
\hline $1 f / r$ & p & $\Lambda \Delta / V$ & rf & اتثاق امور ادارى و مديرتى & & \\
\hline$\Delta V / I$ & 18 & $P r / q$ & ir & حمام و دستشويى در اتثاق كاركنان & & \\
\hline
\end{tabular}




\begin{tabular}{|c|c|c|c|c|c|c|}
\hline \multicolumn{7}{|c|}{ وضعيت طراحى فراتير آسايشكاهها } \\
\hline \multicolumn{2}{|c|}{ عدم رعايت } & \multicolumn{2}{|c|}{ رعايث } & & & \\
\hline ورود & تعداد & توصد & تعداد & & & \\
\hline TNG & $\wedge$ & $n / f$ & $r \cdot$ & وجود يلههاى مناسب & & \\
\hline$e g / p^{\circ}$ & ir & $\Delta r / \varepsilon$ & 10 & وجود نردهاي كمكي يله & & \\
\hline$A T / \Lambda$ & $m$ & IV/a & $\Delta$ & سطج شيبدار منادب & يله و سطح شيبدار & \\
\hline$A T / \Lambda$ & $m$ & $1 W / 9$ & $\Delta$ & وجود نردهاي كمكى سطح شيبدار & & \\
\hline ta & $\checkmark$ & $\checkmark \bowtie$ & r) & قابل تشخيص:بودن رئك در & & \\
\hline V/I & r & $9 Y / 9$ & re & مناسببودن عرض در & & \\
\hline p/e & 1 & 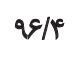 & rV & آستائهنداشتن در & & \\
\hline$\Delta$. & if & $\Delta$. & if & دستكيره هناسب & & \\
\hline$v / 1$ & $r$ & $9 Y / 9$ & q & عرض ورودى مناسب & & شاخصهاي تعريفشلشه \\
\hline IV/a & $\Delta$ & $A Y /$ & m & أندازه داخلى كاقي & & در زيرمجموعلهاي \\
\hline$r q / r$ & 11 & $9 \cdot / V$ & iv & وجود ميلههاى كمكى & & \\
\hline 10 & rA & • & • & وجود سيستم آزير خطر مناسب & 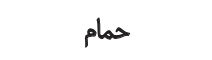 & \\
\hline $1 \in / 4$ & f & $\Lambda \Delta / N$ & $M$ & شيرهاى مناسب آب & & \\
\hline $1 . / N$ & r & $19 / r$ & ro & غير لغزندهبودن كف حمام & & \\
\hline IV/a & $\Delta$ & $A Y / \Lambda$ & m & تعداد مناسب حمام & & \\
\hline TA & $\checkmark$ & VQ & M & ابعاد مناسب حياط & & \\
\hline$\Delta r / s$ & 10 & $r g / 4$ & $\mathbb{1 r}$ & المكان دسترسى أسان به حياط & حhe & \\
\hline$r T / 9$ & ir & $\Delta V / /$ & 19 & وجود صندلى و نيمكت در حياط & & \\
\hline$r e / f$ & ir & $\Delta \Psi / \varepsilon$ & 10 & وجود باغجهه مناسب براى باغباني & & \\
\hline
\end{tabular}

年

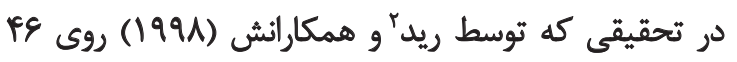
فرد مسن قبل و بعد از ورودشان به به آسايشكاه انجام كرفته

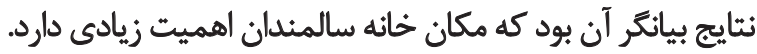

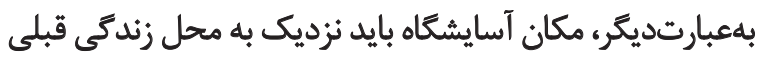

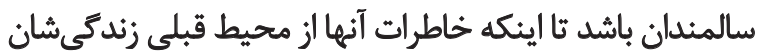

جدا نشود و روابط آنها با دوستان و خانواده حفظ شود [IF] در مطالعه حاضر، بناى آسايشكاهها از نظر ايثكه آسايشكاه

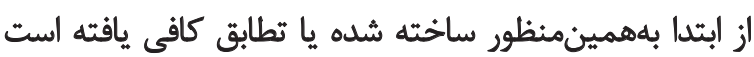
و نيز از نظر دوربودن مكان آسايشكاه از كارخانهانها، انبارها،
آسايشعاههاى بازديدشده اساساً براى كاربرى فعلى ساخته نشده

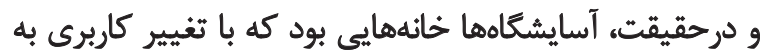

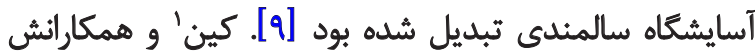

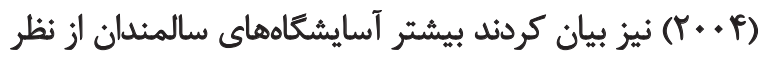

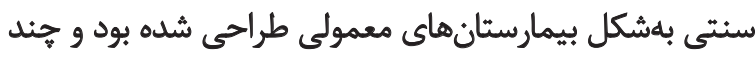

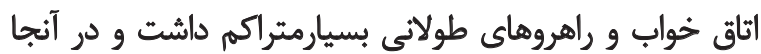

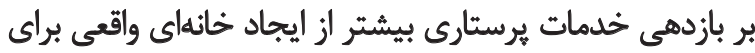

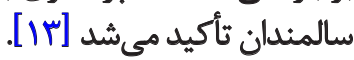




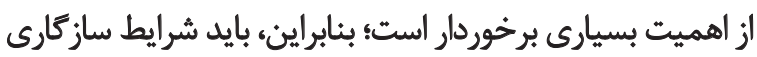

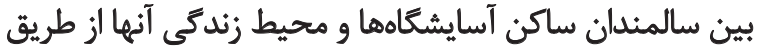

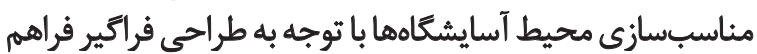
شود تا بر توان و سازكارى و كيفيت زندئى آنان بيافزايد. توجه به مقوله طراحى فراكير در محيط آسايشعاه سالمندان

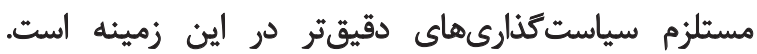

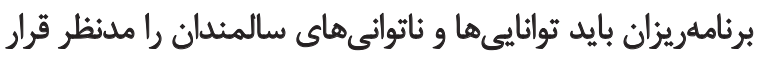

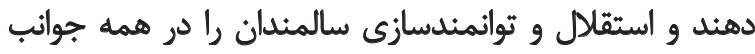

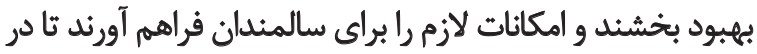

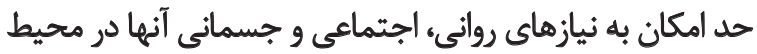

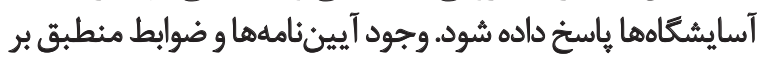

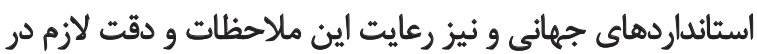

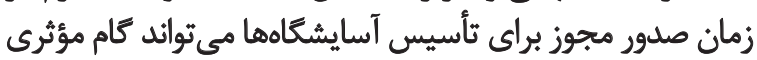

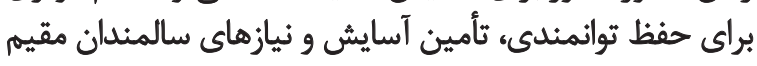
آسايشعاهها باشيد.

$$
\text { تشيكر و قدرواني }
$$

درانتها از مسئولان محترم دانشكاه علوم بهزيستى و ثوانبخشى مانى

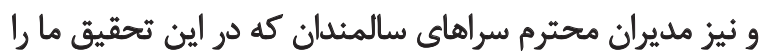
يارى كردند، كمال تشكر و قدردانى بهعمل مي آيد.
خيابانهاي اصلى و يررفتوآمد، مكان بازى بجهها، مسطحبودن

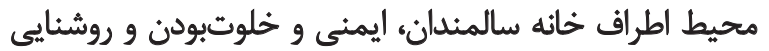

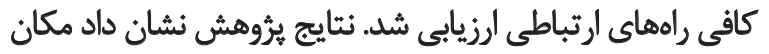

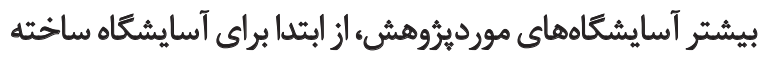

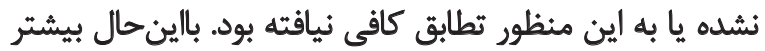

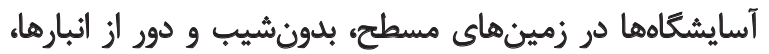

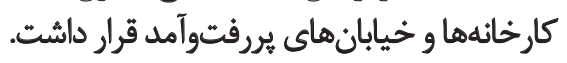

انجمن ملى تحقيقات مسكن آمريكا" تحقيقى درباره بررسى

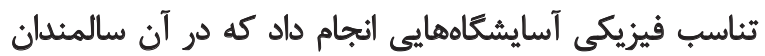

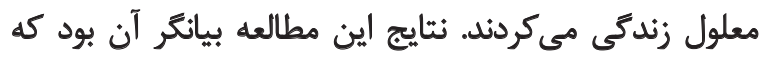

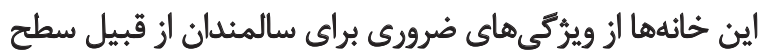

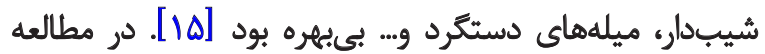

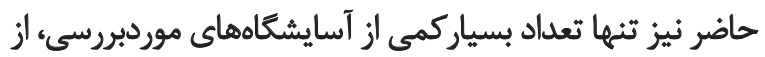

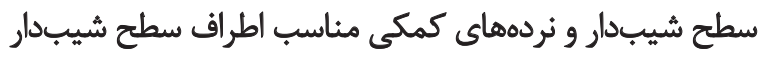
برخوردار بود. - مبدار

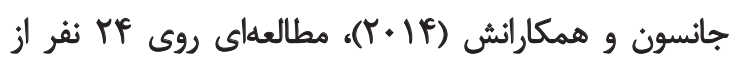

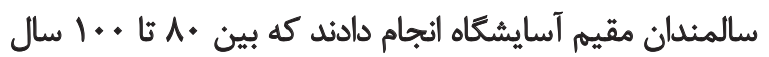

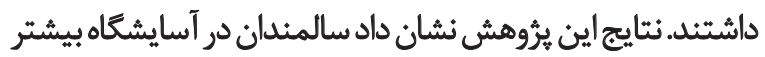

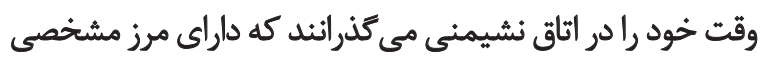

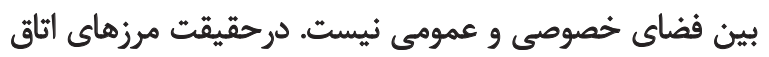

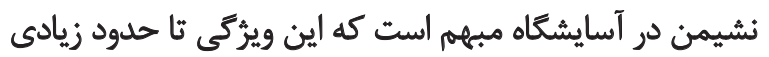

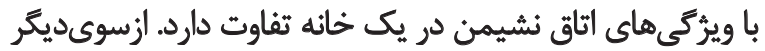

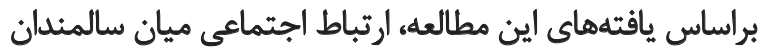
در اتأ نشيمن ضعيف و دجار اضمحلال است التهائ

برهميناساسء جانسون و همكارانش معتقديند اتاق نشيمن

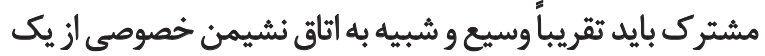

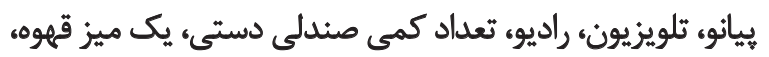

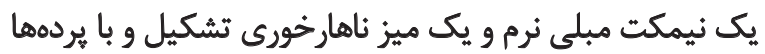

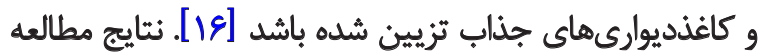

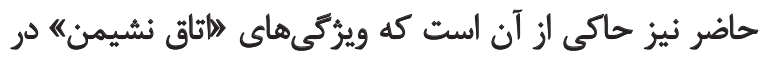

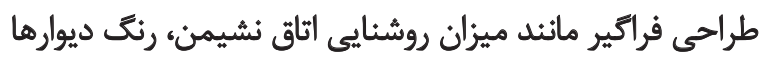

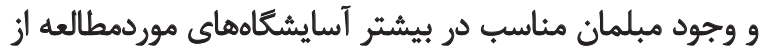

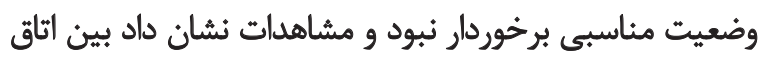

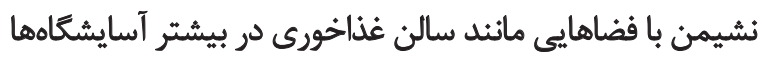
مرز مشخصي وجود نداشت.

\section{تيجيكيرى نمهايى}

يافتههاى اين يُوهش نشان داد بيشتر آسايشكاههاى سالمندان

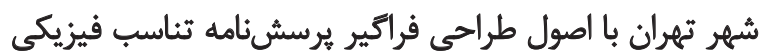

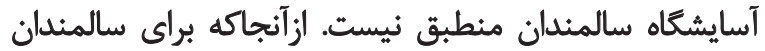

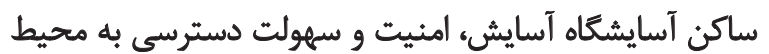

3. National American housing survey 


\section{References}

[1] Hsueh MN, Yeh ML. A conceptual analysis of the process of empowering the elderly at the community level. Hu li za zhi (Journal of Nursing). 2006; 53(2):5-10.

[2] Rashedi V, Gharib M, Yazdani AA. Social participation and mental health among older adults in Iran. Iranian Rehabilitation Journal. 2014; 12(19):9-13.

[3] Ghazi K, Foroughan M, Hosseini M, Hosseinzadeh S, \& Askari, M. [The client satisfaction of delivered services in private nursing homes for elderly: a survey in the Provinces Of Golestan, Mazandaran, Semnan and Northern Khorasan in 2012 (Persian)]. Journal Of Sabzevar University Of Medical Sciences. 2013; 20(3):320-330.

[4] Habibi A, Nikpour S, Seiedoshohadaei M, Haghani H. [Quality of life and status of physical functioning among elderly people in west region of Tehran: a cross-sectional survey (Persian)]. Iran Journal of Nursing. 2008; 21(53):29-39.

[5] Fathi M, Yaghmayi F, Shahsavari S. [Daily living activities of the elderly living in nursing homes Kurdistan (Persian)]. Journal of Nursing and Midwifery, Shahid Beheshti University of Medical Sciences and Health Services. 2008; 62:20-26.

[6] Rashedi V, Rezaei M, Gharib M. Prevalence of cognitive impairment in community-dwelling older adults. Basic and clinical Neuroscience. 2014; 5(1):28-30

[7] Hesamzadeh A, Mohammadi F, Fallahi Khoshknab M, Rahgozar M. [Comparison of elderlys' quality of life living at homes and in private or public nursing homes (Persian)]. Iranian Journal of Ageing. 2010; 4(14):66-74

[8] Nazari SH, Rashedi V, Mohammadi H, Yousefi M. [Relationship between cognitive status and activities of daily living among the elderly of nursing homes (Persian)]. Journal of Kermanshah University of Medical Sciences. 2015; 18(12):744-46

[9] Nozari Sh. [The design guidelines of residential open spaces with regard to vision problems in elderly people (Persian)]. Soffeh. 2004; 39:45-66.

[10] Hosseini FS, Hoseinzadeh R. [The effect of physical activity on physical and mental health in older men (Persian)]. Journal of Health and Care. 2012; 13(2):19-25.

[11] Minemawari Y, Kato T, Aso K. Cognitive function and basic activity of daily living of elderly disabled inpatients. Japanese Journal of Geriatrics. 2000; 37(3):225-32.

[12] Abdollahi F, Mohammadpour RA. [Health related quality of life among the elderly living in nursing home and homes (Persian)]. Journal of Mazandaran University of Medical Sciences. 2013; 23(104):20-25.

[13] Kane RA, Kane RL, Bershadsky B, Cutler LJ, Giles K, Liu $\mathrm{JJ}$, et al. Measures, indicators, and improvement of quality of life in nursing homes. Pittsburgh: University of Pittsburgh; 2004.

[14] Blake KS, Simic A. Elderly housing consumption: Historical patterns and projected trends. Virginia: ICF Consulting Inc; 2005.

[15] Crews DE, Zavotka S. Aging, disability, and frailty: implications for universal design. Journal of Physiological Anthropology. 2006; 25(1):113-18.
[16] Jonsson O, Östlund B, Warell A, Dalholm Hornyánszky E. Furniture in Swedish nursing homes: a design perspective on perceived meanings within the physical environment. Journal of Interior Design. 2014; 39(2):17-35. 
